\title{
Moving NRQCD
}

\section{G.M. von Hippel* (for the HPQCD collaboration)}

Institut für Kernphysik, Johannes-Gutenberg-Universität Mainz, 55099 Mainz, Germany

Email: hippel@kph.uni-mainz.de

Theoretical predictions for exclusive semileptonic decays of heavy mesons are essential for determining CKM matrix elements and constraining new physics.

On the lattice, one must treat the heavy quark in an effective theory, as $1 / M \ll a$. At high recoil, discretization errors from the large 3-momentum of the final meson in the rest frame of the heavy meson are significant.

A technique to avoid these errors is to give the heavy meson a significant "external" momentum in the lattice frame. The decay meson momentum is then not large on the lattice and a wider $q^{2}$ region can be covered. While boosting to a moving frame increases the discretization error for the quarks in a $B$ meson by $\propto\left(\gamma^{2}-1\right) \Lambda_{\mathrm{QCD}}^{2}$, the discretization error of the final-state light meson behaves like $\propto\left(\frac{1}{2}\left|\boldsymbol{p}_{F}\right|\right)^{2}$, and using $q^{2}=\left(p_{B}-p_{F}\right)^{2}$, one can pick an optimal $v$ to minimize the total error.

The key fact in applying the boosted frame technique is that almost all of the heavy meson momentum is carried by the heavy quark whilst the dynamics of the heavy quark inside the heavy meson remain non-relativistic. This leads to a generalization of Non-Relativistic Quantum Chromodynamics (NRQCD) to the case of moving heavy quarks, called moving NRQCD (mNRQCD). We have carried out extensive perturbative and non-perturbative tests of the formalism, which show that the decay constants of both heavy-light and heavy-heavy mesons can be calculated with small systematic errors (although at the cost of increasing statistical errors) up to much larger momenta than with NRQCD.

35th International Conference of High Energy Physics

July 22-28, 2010

Paris, France

\footnotetext{
*Speaker.
} 
Relating the Dirac spinor $\Psi$ to the Pauli spinors $\psi_{v}$ and $\xi_{v}$ for quarks and antiquarks in a frame boosted with the velocity $v$ by a field transformation

$$
\Psi(x)=S(\Lambda) T_{\mathrm{FWT}} e^{-i m u \cdot x \hat{\gamma}^{0}} A_{D_{t}} \frac{1}{\sqrt{\gamma}}\left(\begin{array}{c}
\psi_{v}(x) \\
\xi_{v}(x)
\end{array}\right)
$$

with the spinorial Lorentz boost $S(\Lambda)$, the Foldy-Wouthuysen-Tani transformation $T_{\mathrm{FWT}}$, and a field transformation $A_{D_{t}}$ designed to remove unwanted temporal derivatives from the Lagrangian gives the (continuum, Minkowski-space) Lagrangian [1]

$$
\mathscr{L}=\psi_{v}^{\dagger}[i D_{0}+\underbrace{i \boldsymbol{v} \cdot \boldsymbol{D}+\frac{\boldsymbol{D}^{2}-(\boldsymbol{v} \cdot \boldsymbol{D})^{2}}{2 \gamma m}}_{=H_{0}}+\underbrace{\frac{g}{2 \gamma m} \boldsymbol{\sigma} \cdot \boldsymbol{B}^{\prime}+\mathscr{O}\left(1 / m^{2}\right)}_{\delta H}] \psi_{v}+\left(\begin{array}{c}
\psi_{v} \rightarrow \xi_{v} \\
m \rightarrow-m
\end{array}\right),
$$

where the leading $\mathscr{O}\left(1 / \mathrm{m}^{3}\right)$ term is retained in $\delta H$ to ensure the proper power counting for heavyheavy states; on the lattice, additional terms have to be added for $\mathscr{O}\left(a^{4}, \alpha_{s} a^{2}\right)$ improvement. With partial exponentiation to avoid the well-known parabolic instability, this yields the lattice Lagrangian

$$
\begin{aligned}
\mathscr{L}_{\psi_{v}}(\boldsymbol{x}, \tau) & =\psi_{v}^{+}(\boldsymbol{x}, \tau)\left[\psi_{v}(\boldsymbol{x}, \tau)-K(\tau) \psi_{v}(\boldsymbol{x}, \tau-a)\right] \\
K(\tau) & =\left(1-\frac{\left.\delta H\right|_{\tau}}{2}\right)\left(1-\frac{\left.H_{0}\right|_{\tau}}{2 n}\right)^{n} U_{4}^{\dagger}(\tau-a)\left(1-\frac{\left.H_{0}\right|_{\tau-a}}{2 n}\right)^{n}\left(1-\frac{\left.\delta H\right|_{\tau-a}}{2}\right) .
\end{aligned}
$$

Note that $H_{0}$ is bounded from below at all $v$, as opposed to the case of HQET in a moving frame. Also note that for $v=0, \mathrm{mNRQCD}$ reduces to ordinary NRQCD.

We have computed the wavefunction renormalization constant $Z_{\psi}$, vacuum energy shift $E_{0}$, mass renormalization constant $Z_{m}$, and frame-velocity renormalization constant $Z_{v}$ at the one-loop level [1]; a two-loop calculation is in progress. Since the full $\mathrm{mNRQCD}$ action is very complicated, we employ automated methods (HiPPy/HPsrc [2]) for this purpose. We have also calculated the one-loop matching coefficients for the vector and tensor currents needed for radiative and semileptonic $B$ decays [3].

Going beyond perturbation theory, we have tested non-perturbative improvement [3] to restore cubic symmetry, and have shown that the decay constants of both heavy-light and heavyheavy mesons can be calculated with small systematic errors up to much larger momenta than with NRQCD [1].

In what is intended as the central application of mNRQCD, form factors for $B$ and $B_{s}$ decays, including $B \rightarrow K^{(*)} \ell \ell$ and $B \rightarrow \pi \ell v$, are currently being measured [4] towards low $q^{2}$. Preliminary results suggest that a somewhat larger range in $q^{2}$ may become accessible by using mNRQCD.

\section{References}

[1] R. R. Horgan et al., Phys. Rev. D 80 (2009) 074505 [arXiv:0906.0945].

[2] A. Hart, G.M. von Hippel, R.R. Horgan and E.H. Müller, Comput. Phys. Commun. 180 (2009) 2698 [arXiv:0904.0375].

[3] E. H. Müller et al., PoS LAT2009 (2009) 241 [arXiv:0909.5126].

[4] Z. Liu et al., PoS LAT2009 (2009) 242 [arXiv:0911.2370]; S. Meinel et al., PoS LATTICE2008 (2008) 280 [arXiv:0810.0921]; PoS LAT2007 (2007) 377 [arXiv:0710.3101]. 